\title{
Chewing Well and Being Healthy
}

Majid Nimrouzi ${ }^{1,2,3}$ and Babak Daneshfard ${ }^{1,2,3 *}$

${ }^{1}$ Research Center for Traditional Medicine and History of Medicine, Shiraz University of Medical Sciences, Shiraz, Iran

${ }^{2}$ Essence of Parsiyan Wisdom Institute, Phytopharmaceutical Technology and Traditional Medicine Incubator, Shiraz University of Medical Sciences, Shiraz, Iran

${ }^{3}$ Department of Traditional Persian Medicine, School of Medicine, Shiraz University of Medical Sciences, Shiraz, Iran

\section{Short Communication}

People often look for complex ways to solve our daily problems. We have visited many patients in clinic that resist to accept our simple advices and instead ask for more complex herbal or chemical medicines.

On the other hand, there are many patients with chronic gastrointestinal (GI) and dermal problems who benefit from observing simple health advices. Chewing well, eating in peace, and avoiding simultaneous eating of some foods including meat, dairy products, fruits, and salads are considered as mainstay measures for maintaining health according to traditional Persian medicine (TPM) [1]. For instance, observing such recommendations is the initial stage in managing a serious health issue like infertility [2].

According to TPM sources, there are four stages of food digestion including GI, hepatic, vascular, and in tissue (cellular). The most important part of digestion takes place in the GI tract. GI digestion in turn comprises oral, gastric, and intestinal phases. We usually neglect the oral phase of digestion while it is the most important stage of digestion; any disturbance in the oral phase would naturally disturb the whole process of digestion.

Prophet Mohammed (PBUH) cited that stomach is the house of every disease. It means that all diseases are somehow correlated with eating. Before start eating, we should keep several considerations in our minds if we want to maintain our health:

- Start eating with true appetite and avoid junk food eating

- Eat one type of food in each meal

- Avoid eating when doing other jobs

- Do not start eating when you are excited or nervous

- Do not start eating after intercourse and heavy exercise

- Chew well (33 times for tough morsels)

- Find peaceful places far from heavy noises during your meals

- Do not eat when your stomach is full or you do not like that food

Among these eight items chewing well is the key point, however, all the points should be observed. Why do we eat? For pleasure? For passing time? Or for survival? In fact, eating may be for pleasure or passing time but the main reason for eating is survival and certainly not just being alive, but for having a healthy life. Our body needs energy to do the daily works. This energy is provided by consuming food. We have limited source of energy in our body and these sources should be renewed.

The first step of digestion starts in the oral cavity by amylase and lipase. On the other hand, lysozymes of oral cavity disinfect the contaminated food moderately. If someone eats fast and swallow the food, the salivary glands do not have enough time to secrete these beneficial enzymes and the morsel falls down in the stomach without suitable preparation. In long time, it weakens the stomach and decreases the quality of absorbed materials from the small intestine.
Oral digestion which starts with smelling and tasting the food is very important for a healthy life. Any morsel or sip of a drink which is entered in our oral cavity may be harmful or beneficial. The gustatory system through a complex process in accompany with the other organs evaluate the ingested food or sipped drink. The gustatory buds in different parts of tongue and soft palate and pharynx perceive the taste of foods and drinks and in accompany with somatotactile perception and smelling, the flavour is perceived. Even the proprioception perceived through chewing by teeth and the sound of chewing transmitted to the auditory system are involved in the process of gestation and digestion [3]. These systems are hands of the sober system of the body to introduce a safe audible substance to the alimentary system in order to prepare it for a highly efficient optimized healthy eating and drinking. Eating in haste would disable this process and spoil the digestion.

When we chew well and do not swallow the morsel, the proprioception of our teeth and jaw warn the alimentary system about the quality of the food which is flaky, dry, creamy, soft, or crunchy [3]. In accordance with this impulse the regulatory system of our alimentary tract reacts appropriately.

The taste buds perceive the nutrient stimulators and in response endocrine organs and peripheral secretory organs signal to digestive system to be prepared for optimal metabolism through secretion of proper digestive enzymes [3].

Indulgence and eating large meals, especially when it becomes a habit, affect the normal homeostasis. Large excessive meals more than the capacity of the body homeostasis leads to raised amount of insulin secretion due to assault of massive amounts of macronutrients into blood circulation; the process which exhausts the homeostatic system and would result in metabolic syndrome [3].

\section{References}

1. Nimrouzi M, Zare M (2014) Principles of Nutrition in Islamic and Traditional Persian Medicine. J Evid Based Complementary Altern Med 19: 267-270.

2. Daneshfard B, Jaladat AM (2016) Male Infertility and Diet: A Perspective of Traditional Persian Medicine. Galen Med J 5: 103-104.

3. Breslin PA (2013) An evolutionary perspective on food and human taste. Curr Biol 23: R409-R18.

*Corresponding author: Babak Daneshfard, MD, PhD in Traditional Persian Medicine, Imam Hossein Square, School of Medicine, Shiraz University of Medical Sciences, Shiraz, Iran, Tel/Fax: +9807132345145; E-mail: babakdaneshfard@gmail.com

Received December 04, 2016; Accepted January 04, 2017; Published January 06 2017

Citation: Nimrouzi M, Daneshfard B (2017) Chewing Well and Being Healthy. J Mol Biomark Diagn 8: 327. doi: 10.4172/2155-9929.1000327

Copyright: @ 2017 Nimrouzi M, et al. This is an open-access article distributed under the terms of the Creative Commons Attribution License, which permits unrestricted use, distribution, and reproduction in any medium, provided the original author and source are credited. 